\title{
Efficient TALEN-mediated gene knockin at the bovine Y chromosome and generation of a sex-reversal bovine
}

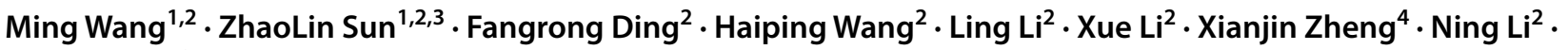 \\ Yunping $\mathrm{Dai}^{2}{ }^{2} \cdot$. Changxin $\mathrm{Wu}^{1}$
}

Received: 26 June 2020 / Revised: 5 May 2021 / Accepted: 14 May 2021 / Published online: 28 May 2021

(c) The Author(s) 2021

\begin{abstract}
Functional elucidation of bovine Y-chromosome genes requires available genome editing technologies. Meanwhile, it has yet to be proven whether the bovine Sry gene is the main or single factor involved in the development of the male phenotype in bovine. Here, we efficiently knocked out four Y-linked genes (Sry, ZFY, DDX3Y, and EIF2S3Y) in bovine fetal fibroblasts (BFFs) with transcription activator-like effector nucleases (TALENs) individually. Furthermore, we used TALENmediated gene knockin at the Sry gene and generated a sex-reversal bovine by somatic cell nuclear transfer (SCNT). The resulting bovine had only one ovary and was sterile. We demonstrate, for the first time, that the Sry gene is an important sex-determining gene in bovine. Our method lays a solid foundation for detecting the biology of the bovine Y chromosome, as it may provide an alternative biological model system for the study of mammalian sex determination, and new methods for the practical application in agricultural, especially for sex predetermination.
\end{abstract}

Keywords Bovine $\cdot$ Y chromosome $\cdot$ TALENs $\cdot$ Knockout $\cdot$ Knockin $\cdot$ Sry gene $\cdot$ Sex reversal

Abbreviations
$\begin{array}{ll}\text { TALENs } & \text { Transcription activator-like effector nuclease } \\ \text { BFF } & \text { Bovine fetal fibroblast } \\ \text { SRY } & \text { Sex determining region Y } \\ \text { ZFY } & \text { Zinc finger protein, Y-linked } \\ \text { DDX3Y } & \text { Asp-Glu-Ala-Asp box polypeptide 3, Y-linked }\end{array}$

ZhaoLin Sun

sunzhaolin@bjsnzz.com

$\triangle$ Ning Li

ninglcau@cau.edu.cn

$\triangle$ Yunping Dai

YDai@cau.edu.cn

1 College of Animal Science and Technology, China Agricultural University, No. 2 Yuanmingyuan Xilu, Beijing 100193, China

2 College of Biological Sciences, China Agricultural University, No. 2 Yuanmingyuan Xilu, Beijing 100193, China

3 Beijing Capital Agribusiness Future Biotechnology Co, 75 Bingjiaokou Hutong, Ltd 100088, No, China

4 Cattle Breeding Research Institute of Beijing Shunxin Xinyuan Co, 3 Anping Street, LtdShunyi District 101318, No, China
EIF2S3Y Eukaryotic translation initiation factor 2, subunit 3, structural gene Y-linked

SCNT Somatic cell nuclear transfer

WT Wild type

\section{Introduction}

The Y chromosome has important specialized functions in male sex differentiation, spermatogenesis, fertility, and related human health issues and diseases [1]. Manipulating Y-chromosome genes is a direct way to investigate their functions. However, perhaps due to the unique structural features of the $\mathrm{Y}$ chromosome, to date research on the mutation of Y-linked genes has been scarce and has been performed only in mice and rabbits with insertional targeting vectors [2], transcription activator-like effector nucleases (TALENs) [3-5], and CRISPR/Cas9 [6-9]. Large animals, especially bovine, are important model and valuable agricultural animals, and the use of genetic modification to establish an alternative biological model for the study of mammalian sex determination or achieve more precise pre-determine the sex [10], is a popular research topic. Therefore, a comprehensive approach to study the function of Y-linked genes in large animals is needed to fully elucidate the biology of the Y chromosome and improve 
related agricultural applications. To the best of our knowledge, no living large animals with gene knockout or gene knockin on the $\mathrm{Y}$ chromosome have been reported. Although, editing of the Y-chromosome specific gene Sry in pig primary cells through nonhomologous end joining (NHEJ) and loxP knockin has been reported, no individual animals have been generated $[11,12]$. Importantly, whether the Sry gene is an important gene for sex determination in bovine, as it is in mice or rabbits [2], is still unclear.

To establish the application of TALEN-mediated gene editing for the Y chromosome in bovine, the bovine Sry gene was chosen as the target gene as the sequence and the expression of the bovine Sry gene has been described [13], and the gene has been reported to be a single-copy gene on the Y chromosome [14]. Importantly, the Sry gene serves as a main genetic switch for male sex development in mice and human [1], and well-defined function in testicular determination in mice and rabbits [2], and the Sry gene is also expressed in the bovine sperm [15], therefore, this locus may be an ideal site for gene knockin to ensure the expression of gene elements of interest on the Y chromosome, and to establish simpler, better systems for sexing sperm, such as the transgene-based sexing system [16].

Here, we report, for the first time, the use of TALEN-mediated gene editing to efficiently knock out Y-linked genes in bovine fetal fibroblasts (BFFs). Notably, efficient knockin of the Sry gene with TALENs was accomplished with a 9-kb dsDNA donor pSRY-EGFP construct containing Thosea asigna virus 2A (T2A) [17] with incorporation of EGFP (T2AEGFP) and the positive selection marker neomycin (NEO) flanked by short homologous arms. Interestingly, while we attempted to use sperm-specific expression of a "suicide" gene to specially ablate the Y-sperm and achieve sex-control, the other dsDNA donor pSRY-DTA containing the "suicide" gene diphtheria toxin fragment A (DTA) gene [18] and driven by the bovine sperm-specific Protamine 1 (bPrm1) promotor [19], was non-precise-homologous recombination (NHR) knockin behind the Sry gene stop codon. Using the NHR-positive geneknockin cells as donor, we generated a viable sex-reversal cow by nuclear transfer. The cow had only one ovary and was sterile. The method lays a solid foundation for detecting the biology of the bovine Y chromosome, as it may provide an alternative biological model system for the study of mammalian sex determination and new methods for practical agricultural applications, especially sex predetermination.

\section{Results}

\section{TALEN-mediated gene targeting in BFFs}

First, we engineered three pairs TALENs directed against the bovine Sry gene instead of using a conventional gene-targeting strategy based on homologous recombination (Fig. 1a). We used a T7 endonuclease I (T7EI) to test the nuclease activity of these TALENs on the Sry gene in BFFs. TALEN pairs 1, 2, and 3 showed gene modification efficiencies of $22.8 \%, 20.6 \%$, and $11.7 \%$, respectively, and the modifications were subsequently verified by TA cloning and sequencing (Fig. 1a). Subsequently, we detected whether the same targeting strategies could be applied to other Y-linked genes and whether the efficiencies for the different genes were different. Zinc finger protein, Y-linked $(Z F Y)$, Asp-Glu-Ala-Asp box polypeptide 3, Y-linked $(D D X 3 Y)$ and eukaryotic translation initiation factor 2, subunit 3, structural gene Y-linked (EIF2S3Y), which were single-copy genes in the male-specific region of the mammalian $\mathrm{Y}$ chromosome in bovine, were chosen [14]. The T7EI assay and sequencing analysis showed that the $Z F Y, D D X 3 Y$, and EIF $2 S 3 Y$ genes could also be efficiently modified by TALENs (Fig. 1b-d). The characteristics of each TALEN pairs are provided in Table $\mathrm{S} 1$.

\section{TALEN-mediated gene knockin in BFFs}

To further establish the applicability of TALEN-mediated editing for the $\mathrm{Y}$ chromosome in bovine, we first produced a TALEN-mediated GFP-knockin allele at the bovine Sry locus. The gene-targeting vector pSRY-EGFP was constructed as shown in Fig. 2a. We introduced Sry TALEN pair 1 , along with a $9 \mathrm{~kb}$ dsDNA donor construct pSRY-EGFP that contained the self-cleaving peptide the $2 \mathrm{~A}$ regions of Thosea asigna virus (T2A) incorporated EGFP (T2A-EGFP) and a positive selection marker Neomycin (NEO) flanked by short homologous arms (843 bp and 910 bp for 5' and 3' arms, respectively) and a negative selection marker diphtheria toxin A (DTA), into the male bovine primary fibroblasts (mBFF1004, XY) (Fig. 2a). After transfection, mBFF1004 cells were selected with G418. As shown in Table S2, we screened 12 single-cell clones and obtained 2 correctly targeted clones by polymerase chain reaction (PCR) genotyping. The primers $\mathrm{P} 1$ and $\mathrm{P} 2$ amplified the expected $2.7-\mathrm{kb}$ band, and the primers $\mathrm{P} 3$ and $\mathrm{P} 4$ amplified the expected 2.2$\mathrm{kb}$ band (Fig. 2b). The PCR products were also identified by sequencing (Fig. 2c, d). The successful GFP-knockin at the Sry locus prompted us to test whether other unique gene elements could be knocked into the Y chromosome-linked gene locus. The donor vector pSRY-DTA, in which the DTA gene was driven by the bovine sperm-specific Protamine 1 (Prm1) promotor, was constructed and was determined to knockin behind the Sry gene stop codon (Fig. 3a). We introduced Sry TALEN pair 1, along with the donor construct that contained the element of interest and a NEO selection marker flanked by short homologous arms (843 bp and $910 \mathrm{bp}$ for 5'and 3'arms, respectively), into mBFF1004 cells (Fig. 3a). After transfection, the mBFF1004 cells were selected with 


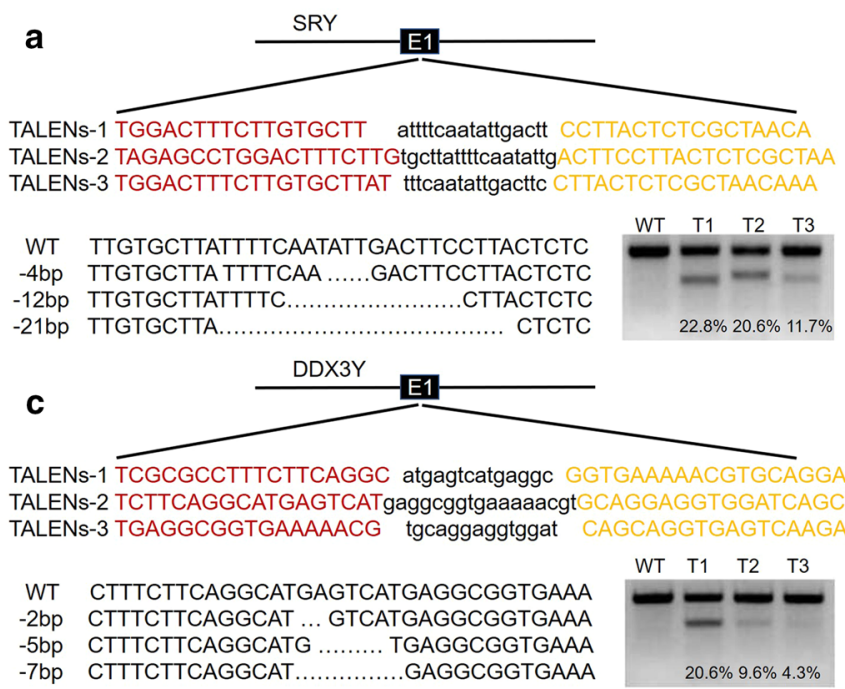

Fig. 1 Design of TALENs for induction of DNA double-strand breaks (DSBs) in different bovine Y-chromosome genes. a Modification of the bovine Y-chromosome Sry gene by TALENs. The recognition sequences for the three TALEN pairs used in this study are highlighted: the left recognition sequence is highlighted in red, and the right recognition sequence is highlighted in yellow. Representative sequencing results of the TA clones revealing different indel mutations mediated by TALENs in the target site are shown at the lower left. Representative results of T7EI assays, which were used to detect

G418. As shown in Table S3, we screened 39 single-cell clones and obtained 1 NHR-targeted clone by PCR genotyping. The P3/P4 primer set amplified the expected 2.2$\mathrm{kb}$ band, while the P5/P6 and P4/P7 primers sets amplified the unexpectedly truncated $1.9-\mathrm{kb}$ band and $3.7-\mathrm{kb}$ band, respectively (Fig. 3b). TA cloning and sequencing further confirmed the above results (Fig. 3c, d). Although the sexcontrol vector did not work, the NHR-targeted clone exhibited the editing of the Sry. The editing included deletion of two important fragments (the $179 \mathrm{bp} 5^{\prime}$ untranslated region (5'-UTR) contained the SRY-binding and Spl-binding motifs and the $302 \mathrm{bp}$ opening reading frame (ORF)), inversion of one important fragment (the $91 \mathrm{bp} \mathrm{5'-UTR}$ and $388 \mathrm{bp}$ ORF containing the HOM box), and a 13 bp extra base insertion (Fig. S1, S2). As the NHR-targeted clone exhibited partial deletion and universal Sry, it would help us to understand the function of the Sry gene in bovine. Therefore, that cell clone was used to perform nuclear transfer. As shown in Table 1, we produced a total of 1578 reconstructed embryos from clone $35 \#, 25.8 \%$ of which successfully entered the blastocyst stage.

\section{Generation of a genetically engineered bovine via somatic cell nuclear transfer (SCNT)}

In total, we transferred 100 transgenic cloned blastocysts into 52 recipients, and a total of two cloned calves were born.

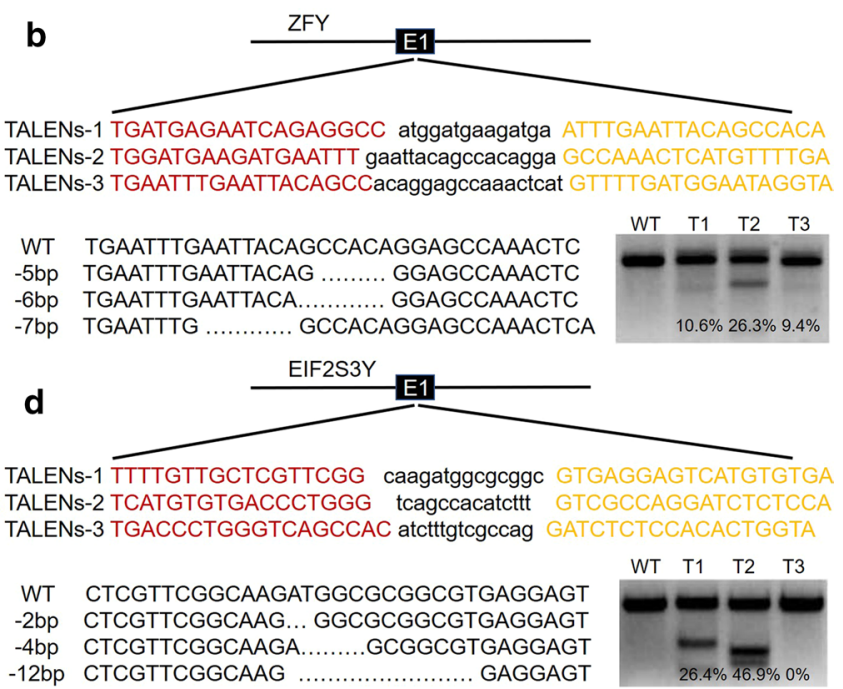

the mutation efficiencies of different TALEN pairs, are shown at the lower right. The mutation frequencies (\% indels) were calculated by measuring the band intensities. E1, Exon 1 of each gene (the TALENtargeting locus); WT, wild-type control cells; T1, TALEN pair 1-transfected cells; T2, TALEN pair 2-transfected cells; T3, TALEN pair 3-transfected cells. b Modification of the bovine Y-chromosome $Z F Y$ gene by TALENs. c Modification of the bovine Y-chromosome $D D X 3 Y$ gene by TALENs. d Modification of the bovine Y-chromosome $E I F 2 S 3 Y$ gene by TALENs

We numbered them by date of birth \#160,517, \#160,705. However, \#160,517 died soon after birth, only \#160,705 remained alive. The calves were positive for gene knockin, as confirmed by PCR genotyping (Fig. 4a) and Southern blot analysis (Fig. 4b). Sex determination by PCR confirmed that the two gene-knockin calves were male (Fig. 4c). Karyotyping of the sex-reversal heifer's cells $(\# 160,705)$ revealed 60 chromosomes, including XY (Fig. 4d). Meanwhile, as shown in Table S4, the expected allele sizes for the eleven bovine-specific microsatellite loci from the sex-reversal heifer \#160,705 were consistent with those of the donor cell line (mBFF1004, XY), confirming that the sex-reversal heifer and the donor cell line (mBFF1004, XY) were from the same genetic source. The sex-reversal heifer \#160,705 was healthy (Fig. 4e). To test for the potential nonspecific mutations induced by the introduction of the TALENs, 10 potential off-target loci of TALEN pair 1 were predicted (Table S5). Sanger sequencing and T7EI analysis were used to determine the off-target effects. The results revealed that no off-target effects occurred in the sex-reversal heifer \#160,705 (Fig. S3).

\section{Phenotype identification of the sex-reversal bovine}

When the heifer \#160,705 was 1 year old, we examined the external genitalia of the sex-reversal heifer. As shown in Fig. 5a, the heifer had female external genitalia. When 

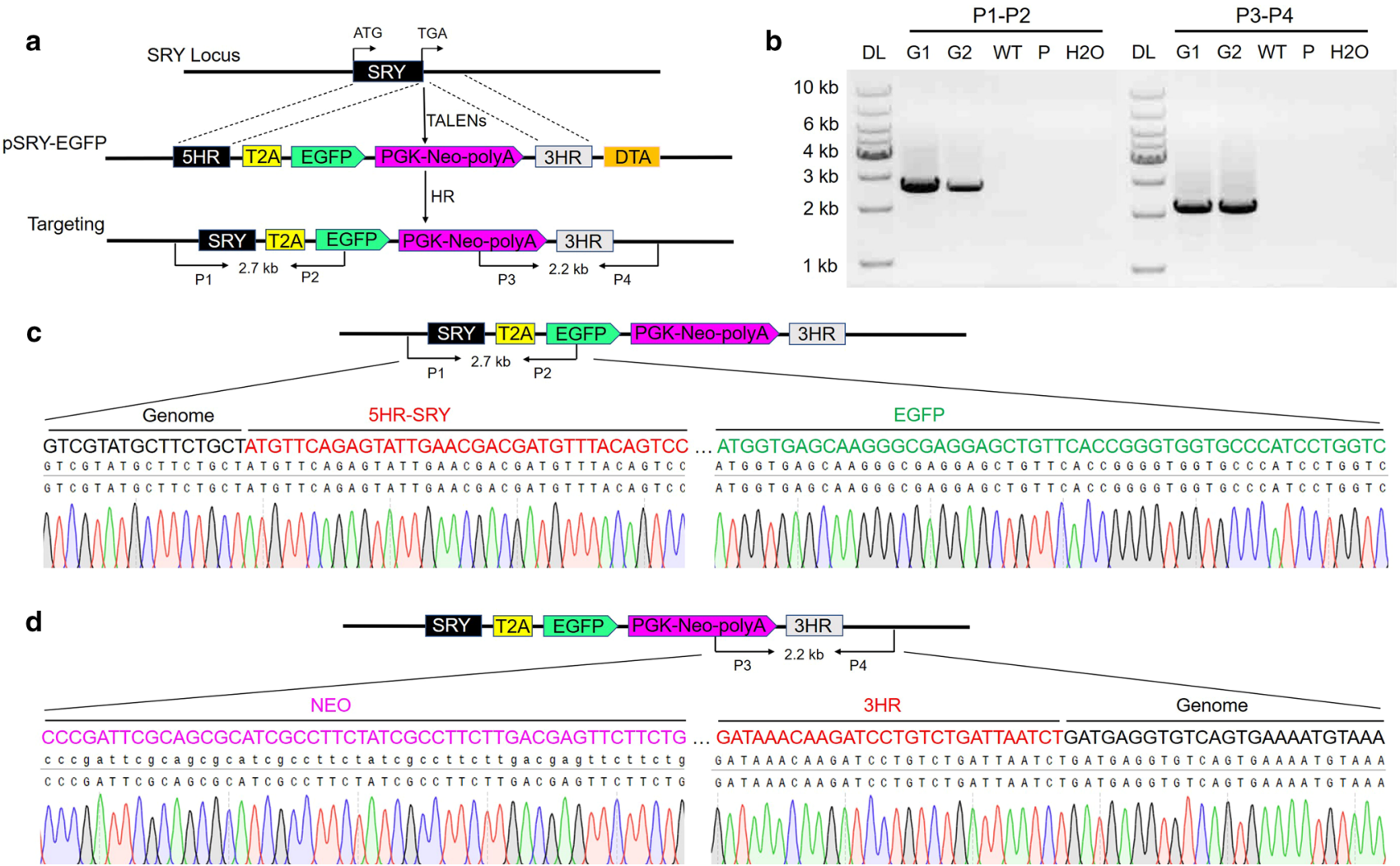

Fig. 2 Production of the pSRY-EGFP-knockin cell clones by TALEN-mediated gene homologous recombination at the Sry locus. a Schematic overview of the strategy used to generate pSRY-EGFPknockin cells in the bovine Sry gene. Sry TALEN pair 1 was used to introduce DSBs. The construct pSRY-EGFP was used as the homologous recombination donor. $\mathrm{P} 1$ and $\mathrm{P} 2$ are the primers outside the $5^{\prime}$ homologous arms; P3 and P4 are the primers outside the 3' homologous arms. b PCR analysis of the pSRY-EGFP-knockin cells. The primer pairs are shown in Fig. 2a. Targeted inclusion of pSRY-EGFP

\#160,705 was 4 years old, we examined its internal genitalia. Compared with wild-type (WT) cow, the XX cow \#160,629, \#160,705 had significantly smaller genitalia, with thinner uterine horns and underdeveloped ovaries (Fig. 5b). The left ovary was solid with few follicles, while the right ovary was fasciculate and had few ovarian structural features (Fig. 5c). No intact follicles were observed by hematoxylin and eosin (H\&E) staining in the \#160,705 ovary, further verifying the developmental disorder of the ovarian interstitial cells (Fig. S4). The absence of follicles indicated ovulatory dysfunction in sex-reversal cow \#160,705.

\section{Fertility of the sex-reversal bovine}

To test the fertility of the sex-reversal heifer \#160,705, we mated it when it was sexually mature with WT bull in which fertility had been previously confirmed. However, we did not detect pregnancy in \#160,705, meanwhile it had no apparent oestrus or copulatory behavior like that of normal females. resulted in amplification of an expected band of 2.7-kb from P1 and $\mathrm{P} 2$ and an expected band of 2.2-kb from P3 and P4. DL, 1-kb DNA ladder; G1-G2, the pSRY-EGFP knockin-positive cells; P, donor vector; WT, wild-type cells; $\mathrm{H}_{2} \mathrm{O}$, negative control. c Sequencing confirmation of the $5^{\prime}$ junction after targeted integration of pSRY-EGFP cassettes into the bovine Sry gene. d Sequencing confirmation of the $3^{\prime}$ junction after targeted integration of pSRY-EGFP cassettes into the bovine Sry gene

The animal did not become pregnant even after injection with the ovulation-stimulating hormones follicle-stimulating hormone (FSH) and luteinizing hormone (LH) before mating. In addition, we tried to obtain fertilized eggs by in vitro fertilization (IVF) using the ovum pick-up (OPU) method; unfortunately, oocytes could not be obtained. We concluded that the infertility of the sex-reversal cow was due to the abnormal development of the ovaries. This finding was consistent with those of previous studies, suggesting that infertility was a common symptom of male-to-female sex-reversal syndrome [20].

\section{Hormone levels in the sex-reversal bovine}

Sex hormones play a crucial roles in follicle formation, embryo implantation and pregnancy. To examine whether the sex-reversal heifer had endocrine features characteristic of females, the levels of six sex hormones were tested using ELISA. The testosterone (T) levels in \#160,705 


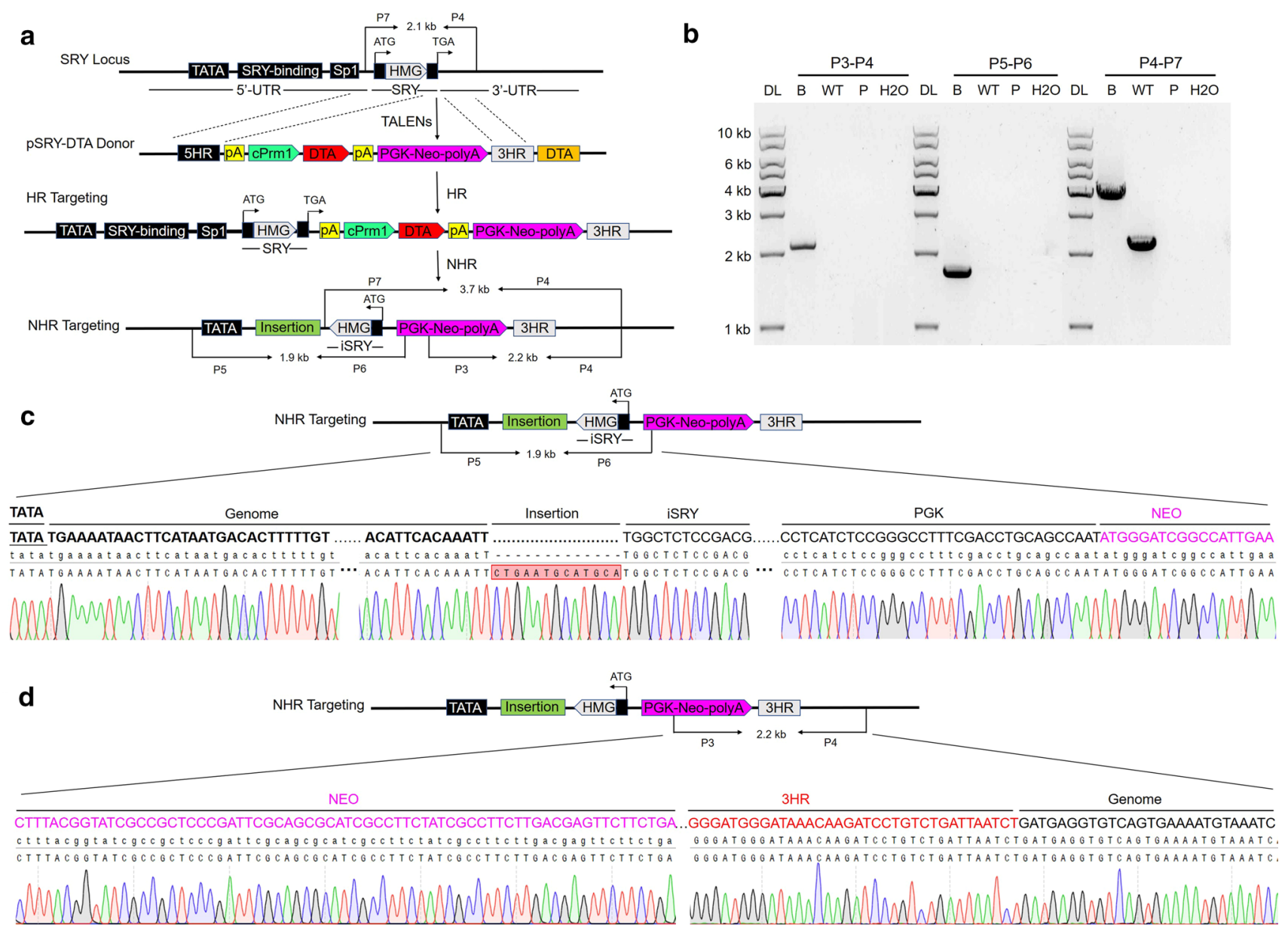

Fig. 3 Production of the pSRY-DTA-knockin cell clones by TALENmediated gene nonhomologous recombination at the Sry locus. a Schematic overview of the strategy used to generate pSRY-DTAknockin cells in the bovine Sry gene. Sry TALEN pair 1 was used to introduce DSBs. The construct pSRY-DTA was used as the homologous recombination donor. P5 and P6 are the primers outside the $5^{\prime}$ homologous arms; P3 and P4 are the primers outside the $3^{\prime}$ homologous arms. P4 and P7 are the primers outside the $5^{\prime}$ and $3^{\prime}$ homologous arms. b PCR analysis of the pSRY-DTA-knockin cells. The primer pairs are as shown in Fig. 3a. Targeted inclusion of
pSRY-DTA resulted in amplification of an unexpected band of 1.9$\mathrm{kb}$ from $\mathrm{P} 5$ and $\mathrm{P} 6$, an expected band of 2.2-kb from P3 and P4, and an unexpected band of 3.7-kb from P4 and P7. Using P4 and P7 also amplified a 2.1-kb band in WT cells. DL, 1-kb DNA ladder; B, the pSRY-DTA non-precise-homologous recombination (NHR) knockinpositive cells; $\mathrm{P}$, donor vector; WT, wild-type cells; $\mathrm{H}_{2} \mathrm{O}$, negative control. (c) Sequencing confirmation of the 5 ' junction after targeted integration of pSRY-DTA cassettes into the bovine Sry gene. (d) Sequencing confirmation of the $3^{\prime}$ junction after targeted integration of pSRY-DTA cassettes into the bovine Sry gene

Table 1 Summary of the nuclear transfer results

\begin{tabular}{llllllll}
\hline No. of cell clone & Oocytes & Reconstruct embryos & Blastocysts & $\begin{array}{l}\text { Blastocysts } \\
\%\end{array}$ & Recipients & Pregnancy at day 60 & Live-born calves \\
\hline$\# 35$ & 2910 & 1578 & 407 & $25.8 \%$ & 52 & 18 & 1 \\
\hline
\end{tabular}

were significantly lower than those in the WT XY controls and were in the range of those in the WT XX controls (Fig. 5d). Reduced levels of estradiol (E2), progesterone (P), and luteinizing hormone ( $\mathrm{LH})$, which could stimulate the development and maintenance of female reproductive tissues, were detected in the $\# 160,705$ (Fig. 5g-i).
However, elevated levels of prolactin (PRL) and folliclestimulating hormone (FSH) were observed in \#160,705 (Fig. 5e, f). These results suggested that the ovarian developmental abnormalities might have resulted in aberrant levels of sex hormones, leading to sterility in sex-reversal cow \#160,705. 

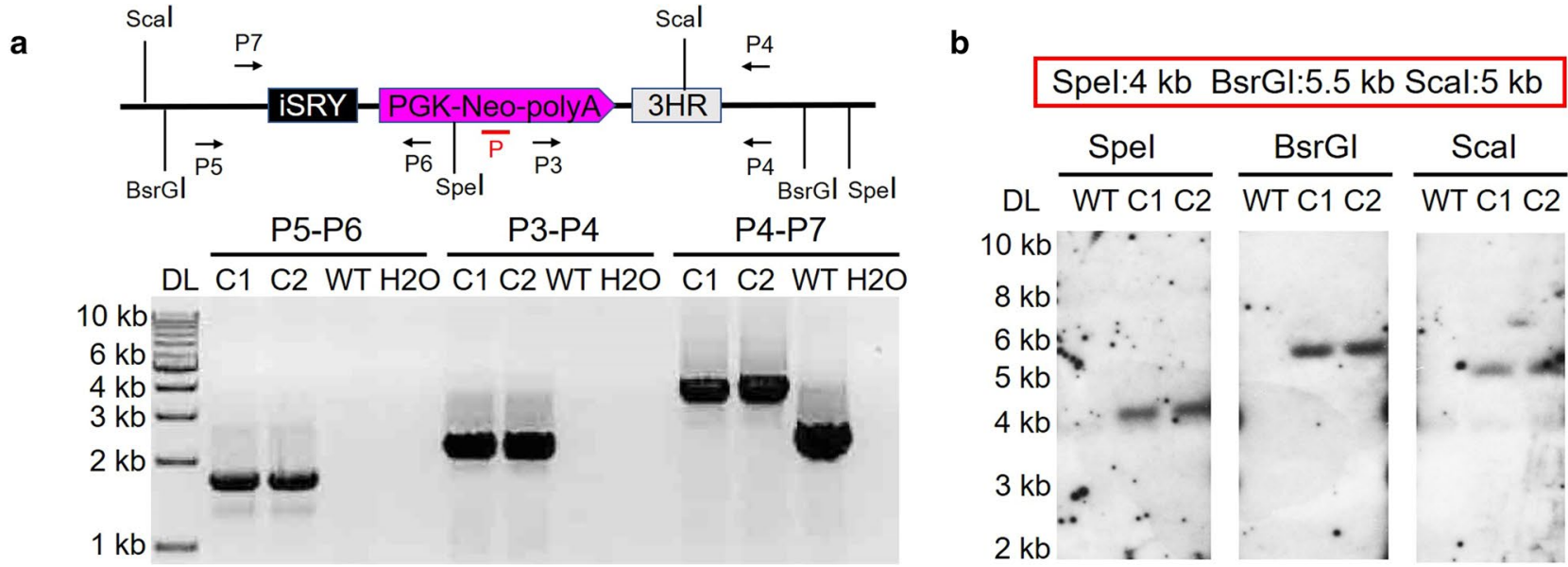

c

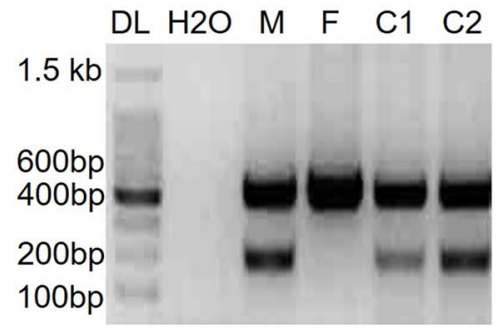

d

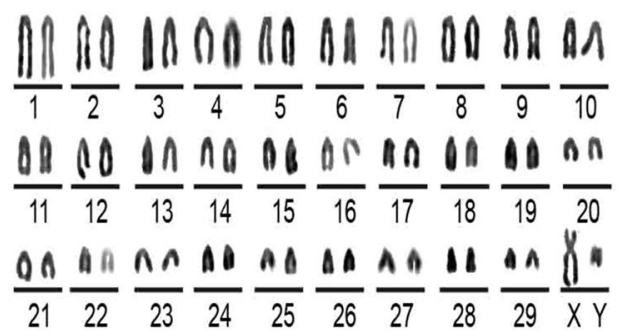

e

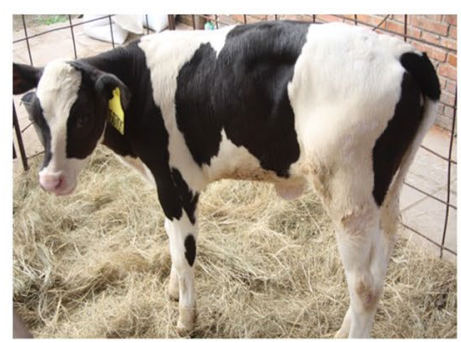

Fig. 4 Generation of sex-reversal bovine by TALEN-mediated gene homologous recombination at the Sry locus. a Schematic of the analysis of the pSRY-DTA-knockin bovine. The primers P5 and P6 amplified a $1.9-\mathrm{kb}$ product, the primers P3 and P4 amplified a 2.2$\mathrm{kb}$ product, and the primers $\mathrm{P} 4$ and $\mathrm{P} 7$ amplified a $3.7-\mathrm{kb}$ product to confirm positive pSRY-DTA-knockin cloned bovine. DL, 1-kb DNA ladder; C1 and C2, the pSRY-DTA NHR knockin-positive heifers (C1 represents \#160,517, and C2 represents \#160,705); WT, wild-type bull; $\mathrm{H}_{2} \mathrm{O}$, negative control. The red line represents the DNA probe used for Southern blot analysis. The restriction endonucleases SpeI, BsrGI, and ScaI were used for Southern blot analysis. b Southern blot analysis of the gene-knockin bovine. The red rectangular box represents the positive band size obtained using different enzymes. Upon digestion with SpeI, a band of $4.0-\mathrm{kb}$ resulting from targeted inclu-

\section{Discussion}

In this study, we found that Y-linked genes can be efficiently targeted by TALEN-mediated gene-editing strategies in BFFs. In addition, we successfully established a TALENmediated method for efficient long dsDNA donor knockin in the Sry locus of the bovine Y chromosome and generated a viable bovine with sex reversal. For the first time, we used TALENs to edit Y chromosome-linked genes such as Sry, $Z F Y, D D X 3 Y$, and EIF2S3Y in bovine, and the highest efficiency for different TALEN pairs were $22.8 \%, 26.3 \%, 20.6 \%$, and $46.9 \%$, respectively. The editing efficiency for the Sry gene was similar to that in mouse embryonic stem (mESCs) cells [3]. This finding proves that TALENs can be applied for bovine Y chromosome-linked genes editing. This method sion of pSRY-DTA was detected. Using the BsrGI, the expected fragment size was 5.5-kb; using the ScaI, the expected fragment size was 5.0-kb. $\mathrm{C} 1$ and $\mathrm{C} 2$, the pSRY-DTA NHR knockin-positive heifers (C1 represents \#160,517, and C2 represents \#160,705); WT, wild-type bull. c Sex determination by PCR method. The couple primers were used to amplify the bovine genomic DNA. For bulls, two fragments of 300-bp and 538-bp were amplified from the Y-specific primer and a bovine-specific primer, while for heifers, a clear single band of 538-bp was amplified. DL, 100-bp DNA ladder; C1, sex-reversal heifer \#160,517; C2, sex-reversal heifer \#160,705; M, wild-type bull; F, wild-type heifer; $\mathrm{H}_{2} \mathrm{O}$, negative control. d Representative image of the XY karyotype of sex-reversal heifer C2 (\#160,705). e Image of sex-reversal heifer C2 (\#160,705)

is expected to aid in elucidation of the biological function of the Y chromosome. Recently CRISPR/Cas9 technology has been widely used to generate gene-editing animals and destroys the entire chromosome in mice $[6,7,21]$. Although the use of TALENs is feasible in bovine, it is far inferior to the use of Cas9, and it is difficult to perform multigene manipulation and targeted deletion on the $\mathrm{Y}$ chromosome with TALENs. Therefore, CRISPR/Cas9 technology could be used to further improve livestock Y-chromosome research in the future. Gene knockin on the Y chromosome has a wide range of applications. Y-chromosome gene knockin was first performed in mESCs by TALENs and traditional gene-targeting vectors [3]. However, the gene-knockin efficiency was low (1\%) in the Sry gene. We used the same method to obtain BFF clones with knockin in the Sry gene 
a

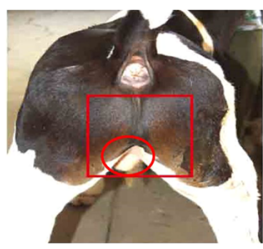

WT XY (Control)

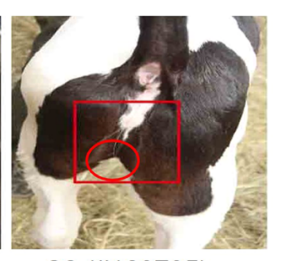

C2 (\#160705) b

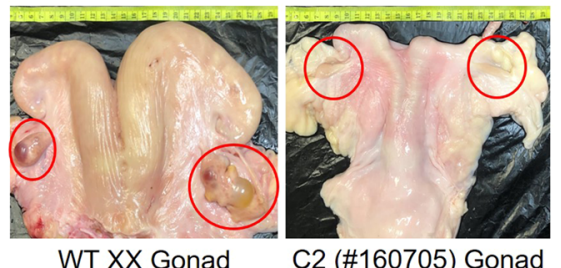

C

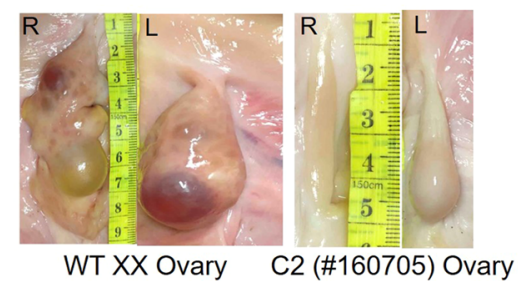

d

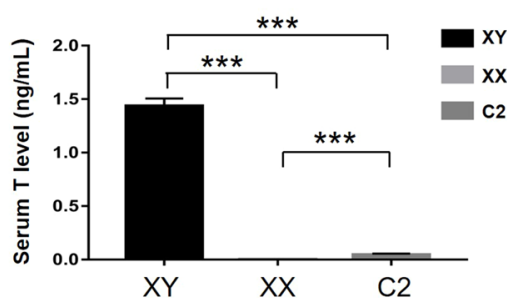

g

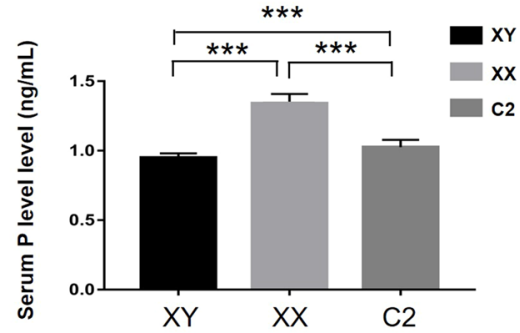

e

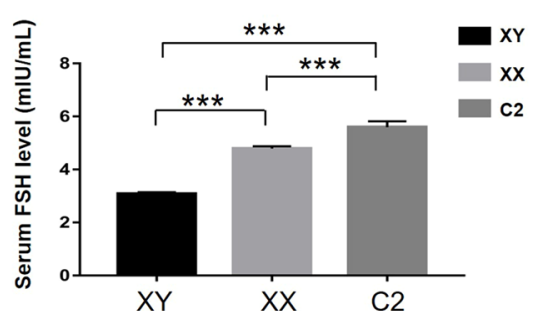

h

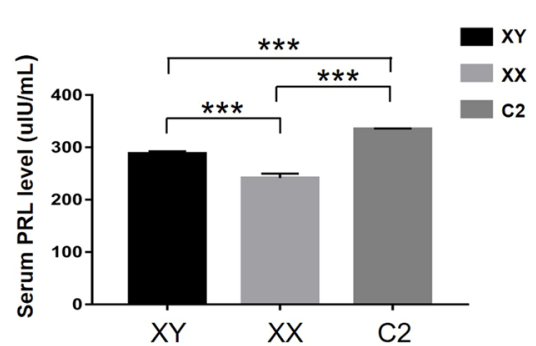

f

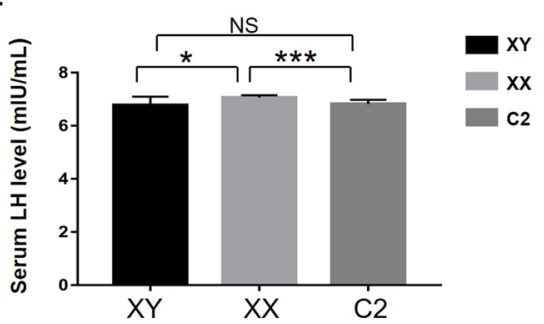

i

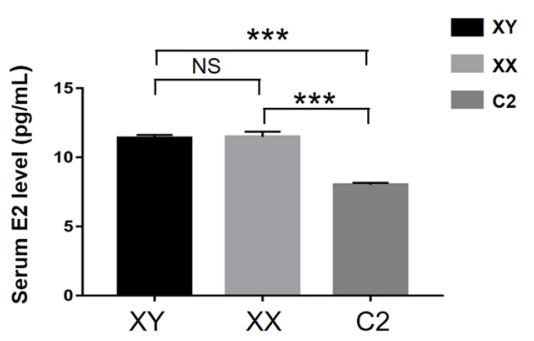

Fig. 5 The ovary and sex hormone detection of the sex-reversal bovine. a Image of the sex-reversal heifer. $\mathbf{b}$ The reproductive organs of the sex-reversal and the WT, XX cow. c The ovaries of the sexreversal and the WT, XX cow. d-i Sex hormones were determined using ELISA. XY, the wild-type bulls; XX, the wild-type heifers; 160,705 , the sex-reversal heifer. The results are presented as the mean \pm SEM. $* p<0.05 ; * * p<0.01 ; * * * p<0.001 ;$ NS, not significant

not be obtained, only one NHR-targeted clone was obtained. This outcome may have been caused by leaky expression of small amounts of sperm-specific DTA in BFFs, consistent with the findings of previous reports [22]. As the NHRtargeted clone exhibited partial deletion and universal Sry, we thought this would help us to understand the function of the Sry gene in bovine. Therefore, we continued cloning and verify whether the Sry gene is the sex-determining gene in bovine from the individual level of animals. Using the NHRtargeted clone, we generated a bovine with Sry gene editing that displayed sex reversal, consistent with the outcomes of mouse and rabbit research. Our bovine with sex reversal had only one ovary and was infertile, which is consistent with finding in mice. Our findings suggest that the Sry gene is the main factor in the development of the male phenotype in bovine. Although we did not detect off-target mutations in any of the potential off-target sites in our sex-reversal bovine using DNA sequencing and T7E1 assay, however, off-target effects could not be ruled out. As a recent study on genetic analyses of a TALEN-mediated gene-knockin hornless bull found that the entire plasmid was unintended inserted into the animals, but this result had not been found in their tive HR-targeted clone containing DTA integration could 
previous studies because the reads of the plasmids were discarded as unmapped, resulting in false negative results [23]. Therefore, the whole-genome sequencing technology can be used for off-target detection, especially HDR plasmid was involved, or the new low off-target Cas 9 system can be used in later studies [24-26].

In summary, we established gene knockout and knockin techniques for the bovine $\mathrm{Y}$ chromosome. Furthermore, we found that the function of the Sry gene is important in male sex determination in bovine. Our results suggest that TALEN-mediated gene editing will enable the study of $\mathrm{Y}$ chromosome biology via genetic manipulation in other agricultural animals and may improve the application of biomedical and agricultural approaches in the future.

\section{Materials and methods}

\section{Animals}

Ovaries from slaughtered mature cows were collected from local abattoir in Beijing, China. Mature control and SCNT recipient cows were obtained from Dairy Cow Center of Beijing. The experimental bovines were housed in stalls with free access to food and water. The bull and the cow of high genetic merit were mated to produce an elite male fetus that was recovered at day 60 . The male bovine fibroblast cell line was isolated from this fetus via disaggregation of all tissue excluding the viscera and limbs, and cultured in Dulbecco's Modified Eagle's Medium (DMEM; Gibco, Grand Island, New York, USA) supplemented with $10 \%$ fetal bovine serum (FBS; Gibco, Grand Island, New York, USA) at $37.5^{\circ} \mathrm{C}$ in an atmosphere of $5 \% \mathrm{CO}_{2}$ and humidified air. The procedures were performed in strict accordance with the Guide for the Care and Use of Laboratory Animals. All the animal work in this study was approved by the Institutional Animal Care and Use Committee of the China Agricultural University under approval number SKLAB-2014-07-05. We performed all surgeries under sodium pentobarbital anesthesia and all attempts were made to minimize animal suffering.

\section{Vector constructs}

The pPGKneoDTA vector was used as the backbone to construct the Sry targeting vector. A 843 bp 5' homologous arm and a 910 bp 3' homologous arm were amplified by PCR from the genome of male BFFs, and cloned into the pPGKneoDTA vector. The T2A-EGFP element and the SV40 polyA-cPrm1-DTA-bGH polyA were synthesized by Sangon Biotech. Conventional molecular cloning and Golden Gate assembly were used to construct the pSRY-EGFP and pSRYDTA vectors. The linearized donor pSRY-EGFP and pSRYDTA were obtained by AhdI digestion. TALEN expression constructs were assembled by Viewsolid Biotech Company (Beijing, China).

\section{Transfection of the primary BFFs}

The male primary BFFs were established as described in the "Animals" section. Then, $8 \mu \mathrm{g}$ TALEN pair 1 and the $4 \mu \mathrm{g}$ linearized pSRY-EGFP or pSRY-DTA donor were nucleofected into $1 \times 10^{6}$ BFFs using Amaxa Nucleofector reagent (Lonza Group AG, Basel, Switzerland) according to the manufacturer's guidelines. The program T-016 was selected. After $48 \mathrm{~h}$, the transfected cells were transferred to $10-\mathrm{cm}$ plates with $10 \%$ FBS containing G418 $(500 \mathrm{mg} / \mathrm{ml})$ at a density of approximately $1 \times 10^{5}$ cells/plate. Individual cell clones were isolated 7-14 days after G418 $(500 \mathrm{mg} / \mathrm{ml})$ selection, expanded, sequenced and cryopreserved after a total of 12-14 days in culture.

\section{T7EI assay}

The editing activity of each TALEN was assayed using T7EI (New England Biolabs, Ipswich, MA, USA) as described previously [27]. Briefly, genomic DNA from TALEN-treated cells was extracted using a DNeasy Blood and Tissue kit (Qiagen, Hilden, Germany). PCR amplicons including nuclease target sites were generated using the following primer pairs: Sry-F/Sry-R for the Sry locus; ZFY-F/ZFY-R for the $Z F Y$ locus; DDX3Y-F/DDX3Y-R for the DDX3Y locus; EIF2S3Y-F/EIF2S3Y-R for the EIF2S3Y locus. All the primers are listed in Table S6. PCR products were then denatured, rehybridized, digested with the T7EI, and analyzed by agarose gel electrophoresis. The mutation frequencies (\% indels) were calculated by measuring the band intensities. The bands were quantified based on the relative band intensities using ImageJ software.

\section{Identification of the positive cell clones by PCR}

To identify the positive cell clones, genomic DNA was extracted from a single cell clone using a DNeasy Blood and Tissue kit (Qiagen, Hilden, Germany). To confirm the successfully edited clones among the pSRY-EGFP-targeted cells, two pairs of primers targeting a sequence between the donor and outside the 5' or 3' homologous arm, respectively, were used: the $\mathrm{P} 1 / \mathrm{P} 2$ primer pair was used for the 5 ' arm and produced a $2.7-\mathrm{kb}$ amplicon, and the $\mathrm{P} 3 / \mathrm{P} 4$ primer pair was used for the 3' arm and produced a 2.2-kb amplicon. To confirm the successfully edited clones among the pSRYDTA-targeted cells, another two pairs of primers between the donor and outside the 5' or 3' homologous arm were used: the $\mathrm{P} 3 / \mathrm{P} 4$ primer pair was used for the $3^{\prime}$ arm and produced a 2.2-kb amplicon, and the primer pair P5/P6 was used for the 5' arm and produced a 1.9-kb amplicon. A primer pair 
targeting a sequence outside the 5' and 3' homologous arms, $\mathrm{P} 4 / \mathrm{P} 7$, which produced a $3.7-\mathrm{kb}$ amplicon, was also used to confirm the successfully edited cell clones. PCR was performed for 35 cycles at $94{ }^{\circ} \mathrm{C}$ for $30 \mathrm{~s}, 60{ }^{\circ} \mathrm{C}$ for $30 \mathrm{~s}$, and $72{ }^{\circ} \mathrm{C}$ for $2-3$ min with a hold at $72{ }^{\circ} \mathrm{C}$ for $10 \mathrm{~min}$. The PCR products were sequenced by TA-Clone. All the primers are listed in Table S6.

\section{SCNT}

SCNT was performed as described previously [28]. Briefly, transgenic cells were transferred into enucleated oocytes for the production of reconstructed embryos in vitro and then fused using the ECM ${ }^{\circledR} 2001$ Electro Cell Manipulation System (BTX, San Diego, CA, USA). The reconstructed embryos were activated with $10 \mathrm{mg} / \mathrm{ml}$ cycloheximide and $2.5 \mathrm{mg} / \mathrm{ml}$ cytochalasin-D in CR1aa medium. Day-7 blastocysts were collected for future transplantation. A total of 100 blastocysts were transferred into 52 recipient cows. For each recipient, we transferred 1-2 transgenic cloned blastocysts. Pregnancy was detected by ultrasonography at 60 days and 180 days post-transfer.

\section{Identification of sex-reversal bovine}

To identify the sex-reversal bovine, genomic DNA was obtained from the ear tissue of potential sex-reversal heifer and WT bull through phenol/chloroform extraction. For PCR analysis, three pairs of primers P3/P4, P5/P6, and P4/P7, were used to confirm successful gene editing in the bovine; these primer sets produced $2.2-\mathrm{kb}, 1.9-\mathrm{kb}$, and $3.7-\mathrm{kb}$ amplicons, respectively. PCR was performed for 35 cycles at $94{ }^{\circ} \mathrm{C}$ for $30 \mathrm{~s}, 60{ }^{\circ} \mathrm{C}$ for $30 \mathrm{~s}$, and $72{ }^{\circ} \mathrm{C}$ for $2-3 \mathrm{~min}$ with a hold at $72{ }^{\circ} \mathrm{C}$ for $10 \mathrm{~min}$. For Southern blot analysis, the genomic DNA $(10 \mu \mathrm{g})$ was digested with the restriction enzymes SpeI, BsrGI, and ScaI, respectively, overnight. A digoxigenin-labeled probe that targeted the NEO gene was amplified using the PCR DIG Probe Synthesis Kit (Roche, Mannheim, Germany) with the primer pair neo-F/ neo-R (Table S6). After $0.7 \%$ agarose gel electrophoresis for 6-8 h, the DNA was transferred to a nitrocellulose filter for blotting. The nitrocellulose membrane (Roche, Mannheim, Germany) was hybridized with a probe for $16 \mathrm{~h}$ and incubated with antibody for $0.5 \mathrm{~h}$. The expected sizes of the positive bands using SpeI, BsrGI, and ScaI were approximately $4.0 \mathrm{~kb}, 5.5 \mathrm{~kb}$, and $5.0 \mathrm{~kb}$, respectively.

\section{Sex determination by PCR method}

Sex determination was performed as previously described [29]. Briefly, multiplex PCR with two sets of PCR primers, Y-chromosome specific primers (BY-F/R), and bovine specific primers (BSP-F/R), was used to test the samples. The amplification reactions were conducted in a total volume of 50 ul using Q5 DNA polymerase (New England Biolabs, Ipswich, MA, USA). The amplification was carried out in a DNA thermal cycler with an initial denaturation at $98^{\circ} \mathrm{C}$ for $5 \mathrm{~min}$ and then 45 cycles at $98{ }^{\circ} \mathrm{C}$ for $20 \mathrm{~s}, 52{ }^{\circ} \mathrm{C}$ for $30 \mathrm{~s}$, and $72{ }^{\circ} \mathrm{C}$ for $30 \mathrm{~s}$ followed by final extension at $72{ }^{\circ} \mathrm{C}$ for $10 \mathrm{~min} .10 \mathrm{ml}$ of each PCR product was electrophoresed on a $2 \%$ agarose gel and stained with ethidium bromide.

\section{Karyotype analysis}

Bovine fibroblasts were used for karyotyping. The fibroblasts were derived from bovine tails that were cut into small pieces and cultured for 7 days. Then, the fibroblasts were incubated with $200 \mathrm{ng} / \mathrm{ml}$ demecolcine (Sigma) for $1 \mathrm{~h}$, resuspended in $0.075 \mathrm{M} \mathrm{KCl}$ at $37{ }^{\circ} \mathrm{C}$ for $10-30 \mathrm{~min}$, and fixed with Carnoy's fixative (25\% acetic acid in methanol) for $30 \mathrm{~min}$. The cells were plated on precleaned slides, and karyotype analysis was performed using VideoTest-Karyo3.1.

\section{Microsatellite analysis}

Microsatellite analysis was performed on the sex-reversal heifer cells and the gene knockin-positive cell clone \#35 based on the protocol for StockMarks ${ }^{\circledR}$ Horse, Cattle, and Dog Genotyping Kits (Applied Biosystems, Foster City, CA, USA). Eleven microsatellite loci located on different bovine chromosomes were first visualized with $3 \%$ agarose gel electrophoresis and further confirmed by capillary gel electrophoresis with fluorescently labeled amplimers and laser scanning using an ABI PRISM $® 3100$ Genetic Analyzer. The amplification results were determined using the Genotyper software plots of GeneScan software. Allele sizes were assigned using Genemapper version 4.0 (Applied Biosystems, Foster City, CA, USA).

\section{Prediction of off-target sites}

The genome sequence (species name: Cow, Latin name: Bos taurus, reference genome version: Ruminant Reference Genome, 4.6.1/bos Tau7) was divided into a small units of $10 \mathrm{~kb}$. The left and right sequences of Sry TALEN pair 1 were input into the Cutadapt linked Adapter program (https:// cutadapt.readthedocs.io/en/stable/guide.html\#linked-adapt ers) with full length matching, no indels allowed, and a maximum base mismatch of 5. Linked Adapter cyclically searched within each segmented small unit; that was, after a successful match, it searched again from the position after the match until another match could not be found. In the matching process, only results with spacing lengths greater than 10 and less than 30 were retained. 


\section{Hematoxylin and Eosin (H\&E) staining}

H\&E staining was performed as previously described [30]. Briefly, the tissues were fixed with $4 \%$ paraformaldehyde for $48 \mathrm{~h}$, embedded in paraffin wax, sectioned onto slides, stained with H\&E and then analyzed by microscopy (Nikon TS100).

\section{Sex hormone assay}

When the sex-reversal heifer \#160,705 was 1 year old, the sex hormone assay was conducted. Sexually mature and in estrus WT-XX heifers $(n=3)$ and WT-XY bulls $(n=3)$, which were of the same age as the experimental heifer, were chosen as control. And the WT-XX heifers $(n=3)$ were without pregnancy. Serum was obtained by precipitation and centrifugation. Sex hormones, including Testosterone (T), Estradiol (E2), Follicle-stimulating hormone (FSH), Luteinizing hormone (LH), Progesterone (P), and Prolactin (PRL), were measured using an ELISA Kit (IBL, Germany). All experiments were repeated three times. The data were expressed as the mean \pm SEM.

\section{Statistical analysis}

Statistical analysis of hormonal data was performed using GraphPad Prism version 5.0 for Windows. One-way ANOVA followed by Tukey's multiple comparison test was used for comparisons between groups with normal distributions. Differences between experimental groups were regarded as significant when $\mathrm{P} \leq 0.05$.

Supplementary Information The online version contains supplementary material available at https://doi.org/10.1007/s00018-021-03855-1.

Acknowledgements We thank Chunjiang Zhao for the analysis of the microsatellite assay. We also thank Liang Ma and Meili Wang for their proficient technical assistance and all the colleagues who gave us valuable suggestions and comments about our experiments and manuscript.

Author contributions ZS, MW, NL, and YD designed the project and planed the experiments. MW, ZS, JW, and FD, performed the experiment. LL, XL, XZ, and HW performed the SCNT. CW provides important suggestions about the manuscript writing. The manuscript was written by ZS and MW. All authors reviewed the manuscript.

Funding This work was supported by the National Transgenic Breeding Project of China (2018ZX08007001).

Availability of data and material All data generated or analyzed during this study are included in this published article and its supplementary information files.

Code availability Not applicable.

\section{Declarations}

Conflict of interest The authors declare no competing financial interests.

Ethics approval The procedures were performed in strict accordance with the Guide for the Care and Use of Laboratory Animals. All the animal work in this study was approved by the Institutional Animal Care and Use Committee of the China Agricultural University under approval number SKLAB-2014-07-05.

Consent to participate Not applicable

Consent for publication Not applicable.

Open Access This article is licensed under a Creative Commons Attribution 4.0 International License, which permits use, sharing, adaptation, distribution and reproduction in any medium or format, as long as you give appropriate credit to the original author(s) and the source, provide a link to the Creative Commons licence, and indicate if changes were made. The images or other third party material in this article are included in the article's Creative Commons licence, unless indicated otherwise in a credit line to the material. If material is not included in the article's Creative Commons licence and your intended use is not permitted by statutory regulation or exceeds the permitted use, you will need to obtain permission directly from the copyright holder. To view a copy of this licence, visit http://creativecommons.org/licenses/by/4.0/.

\section{References}

1. Hughes JF, Page DC (2015) The biology and evolution of mammalian Y chromosomes. Annu Rev Genet 49:507-527. https://doi. org/10.1146/annurev-genet-112414-055311

2. Rohozinski J, Agoulnik AI, Boettger-Tong HL, Bishop CE (2002) Successful targeting of mouse $\mathrm{Y}$ chromosome genes using a sitedirected insertion vector. Genesis 32(1):1-7. https://doi.org/10. 1002/gene. 10020

3. Wang H, Hu YC, Markoulaki S, Welstead GG, Cheng AW, Shivalila CS, Pyntikova T, Dadon DB, Voytas DF, Bogdanove AJ, Page DC, Jaenisch R (2013) TALEN-mediated editing of the mouse Y chromosome. Nat Biotechnol 31(6):530-532. https://doi.org/10. 1038/nbt.2595

4. Kato T, Miyata K, Sonobe M, Yamashita S, Tamano M, Miura K, Kanai Y, Miyamoto S, Sakuma T, Yamamoto T, Inui M, Kikusui T, Asahara H, Takada S (2013) Production of Sry knockout mouse using TALEN via oocyte injection. Sci Rep 3:3136. https://doi. org/10.1038/srep03136

5. Matsubara Y, Kato T, Kashimada K, Tanaka H, Zhi Z, Ichinose S, Mizutani S, Morio T, Chiba T, Ito Y, Saga Y, Takada S, Asahara H (2015) TALEN-mediated gene disruption on Y chromosome reveals critical role of EIF2S3Y in mouse spermatogenesis. Stem cells and development 24(10):1164-1170. https://doi.org/10.1089/ scd.2014.0466

6. Adikusuma F, Williams N, Grutzner F, Hughes J, Thomas P (2017) Targeted deletion of an entire chromosome using CRISPR/ Cas9. Molecular therapy: the journal of the American Society of Gene Therapy 25(8):1736-1738. https://doi.org/10.1016/j.ymthe. 2017.05.021

7. Zuo E, Huo X, Yao X, Hu X, Sun Y, Yin J, He B, Wang X, Shi L, Ping J, Wei Y, Ying W, Wei W, Liu W, Tang C, Li Y, Hu J, Yang H (2017) CRISPR/Cas9-mediated targeted chromosome 
elimination. Genome Biol 18(1):224. https://doi.org/10.1186/ s13059-017-1354-4

8. Song Y, Liu T, Wang Y, Deng J, Chen M, Yuan L, Lu Y, Xu Y, Yao H, Li Z, Lai L (2017) Mutation of the Sp1 binding site in the 5 ' flanking region of SRY causes sex reversal in rabbits. Oncotarget 8(24):38176-38183. https://doi.org/10.18632/oncotarget. 16979

9. Imaimatsu K, Fujii W, Hiramatsu R, Miura K, Kurohmaru M, Kanai Y (2018) CRISPR/Cas9-mediated knock-in of the murine Y chromosomal Sry gene. J Reprod Dev 64(3):283-287. https:// doi.org/10.1262/jrd.2017-161

10. Kurtz S, Petersen B (2019) Pre-determination of sex in pigs by application of CRISPR/Cas system for genome editing. Theriogenology 137:67-74. https://doi.org/10.1016/j.theriogenology. 2019.05.039

11. Carlson DF, Tan W, Lillico SG, Stverakova D, Proudfoot C, Christian M, Voytas DF, Long CR, Whitelaw CB, Fahrenkrug SC (2012) Efficient TALEN-mediated gene knockout in livestock. Proc Natl Acad Sci USA 109(43):17382-17387. https://doi.org/ 10.1073/pnas.1211446109

12. Tan W, Carlson DF, Lancto CA, Garbe JR, Webster DA, Hackett PB, Fahrenkrug SC (2013) Efficient nonmeiotic allele introgression in livestock using custom endonucleases. Proc Natl Acad Sci USA 110(41):16526-16531. https://doi.org/10.1073/pnas.13104 78110

13. Daneau I, Houde A, Ethier JF, Lussier JG, Silversides DW (1995) Bovine SRY gene locus: cloning and testicular expression. Biol Reprod 52(3):591-599. https://doi.org/10.1095/biolreprod52.3. 591

14. Chang TC, Yang Y, Retzel EF, Liu WS (2013) Male-specific region of the bovine $\mathrm{Y}$ chromosome is gene rich with a high transcriptomic activity in testis development. P Natl Acad Sci USA 110(30):12373-12378. https://doi.org/10.1073/pnas.1221104110

15. Li C, Sun Y, Yi K, Li C, Zhu X, Chen L, Zhou X (2011) Detection of the SRY transcript and protein in bovine ejaculated spermatozoa. Asian Australas J Anim Sci 24(10):1358-1364. https://doi. org/10.5713/ajas.2011.11062

16. Harris AF, Nimmo D, McKemey AR, Kelly N, Scaife S, Donnelly CA, Beech C, Petrie WD, Alphey L (2011) Field performance of engineered male mosquitoes. Nat Biotechnol 29(11):1034-1037. https://doi.org/10.1038/nbt.2019

17. Szymczak AL, Workman CJ, Wang Y, Vignali KM, Dilioglou S, Vanin EF, Vignali DA (2004) Correction of multi-gene deficiency in vivo using a single "self-cleaving" $2 \mathrm{~A}$ peptide-based retroviral vector. Nat Biotechnol 22(5):589-594. https://doi.org/10.1038/ nbt957

18. Palmiter RD, Behringer RR, Quaife CJ, Maxwell F, Maxwell IH, Brinster RL (1987) Cell lineage ablation in transgenic mice by cell-specific expression of a toxin gene. Cell 50(3):435-443

19. Jacoues J, Peschon RR, Behringer RL, Brinster RD, Palmiter, (1987) Spermatid-specific expression of protamine 1 in transgenic mice. Proc Natl Acad Sci USA 84(15):5316-5319. https://doi.org/ 10.1073/pnas.84.15.5316
20. Veitia R, Ion A, Barbaux S, Jobling MA, Souleyreau N, Ennis K, Ostrer H, Tosi M, Meo T, Chibani J, Fellous M, McElreavey K (1997) Mutations and sequence variants in the testis-determining region of the Y chromosome in individuals with a 46. XY female phenotype Hum Genet 99(5):648-652. https://doi.org/10.1007/ s004390050422

21. Hsu PD, Lander ES, Zhang F (2014) Development and applications of CRISPR-Cas9 for genome engineering. Cell 157(6):12621278. https://doi.org/10.1016/j.cell.2014.05.010

22. Brockschnieder D, Pechmann Y, Sonnenberg-Riethmacher E, Riethmacher D (2006) An improved mouse line for Cre-induced cell ablation due to diphtheria toxin A, expressed from the Rosa26 locus. Genesis (New York, NY: 2000) 44 (7):322-327. doi:https:// doi.org/10.1002/dvg.20218

23. Young AE, Mansour TA (2020) Genomic and phenotypic analyses of six offspring of a genome-edited hornless bull. Nat Biotechnol 38(2):225-232. https://doi.org/10.1038/s41587-019-0266-0

24. Kleinstiver BP, Pattanayak V, Prew MS, Tsai SQ, Nguyen NT, Zheng Z, Joung JK (2016) High-fidelity CRISPR-Cas9 nucleases with no detectable genome-wide off-target effects. Nature 529(7587):490-495. https://doi.org/10.1038/nature16526

25. Chen JS, Dagdas YS, Kleinstiver BP, Welch MM, Sousa AA, Harrington LB, Sternberg SH, Joung JK, Yildiz A, Doudna JA (2017) Enhanced proofreading governs CRISPR-Cas9 targeting accuracy. Nature 550(7676):407-410. https://doi.org/10.1038/nature24268

26. Tan Y, Chu AHY, Bao S, Hoang DA, Kebede FT, Xiong W, Ji M, Shi J, Zheng Z (2019) Rationally engineered Staphylococcus aureus Cas9 nucleases with high genome-wide specificity. Proc Natl Acad Sci USA 116(42):20969-20976. https://doi.org/10. 1073/pnas. 1906843116

27. Kim HJ, Lee HJ, Kim H, Cho SW, Kim JS (2009) Targeted genome editing in human cells with zinc finger nucleases constructed via modular assembly. Genome Res 19(7):1279-1288. https://doi.org/10.1101/gr.089417.108

28. Wang M, Sun Z, Yu T, Ding F, Li L, Wang X, Fu M, Wang H, Huang J, Li N, Dai Y (2017) Large-scale production of recombinant human lactoferrin from high-expression, marker-free transgenic cloned cows. Sci Rep 7(1):10733. https://doi.org/10.1038/ s41598-017-11462-z

29. Rattanasuk S, Parnpai R, Ketudat-Cairns M (2011) Multiplex polymerase chain reaction used for bovine embryo sex determination. J Reprod Dev 57(4):539-542. https://doi.org/10.1262/jrd. $10-126 \mathrm{~m}$

30. Luo J, Song Z, Yu S, Cui D, Wang B, Ding F, Li S, Dai Y, Li N (2014) Efficient generation of myostatin (MSTN) biallelic mutations in cattle using zinc finger nucleases. PLoS ONE 9(4):e95225. https://doi.org/10.1371/journal.pone.0095225

Publisher's Note Springer Nature remains neutral with regard to jurisdictional claims in published maps and institutional affiliations. 